\title{
Travelling Waves: Interplay of Low to High Reynolds Number and Tan-Cot Function Method to Solve Burger's Equations
}

\author{
Md. Kamrujjaman', Asif Ahmed², Jahrul Alam² \\ ${ }^{1}$ Department of Mathematics, University of Dhaka, Dhaka, Bangladesh \\ ${ }^{2}$ Department of Mathematics and Statistics, Memorial University, St. John's, Canada \\ Email:kamrujjaman@du.ac.bd, ahmedasif.du@gmail.com, alamj@mun.ca
}

How to cite this paper: Kamrujjaman, Md., Ahmed, A. and Alam, J. (2019) Travelling Waves: Interplay of Low to High Reynolds Number and Tan-Cot Function Method to Solve Burger's Equations. Journal of Applied Mathematics and Physics, 7 , 861-873.

https://doi.org/10.4236/jamp.2019.74058

Received: March 7, 2019

Accepted: April 16, 2019

Published: April 19, 2019

Copyright $\odot 2019$ by author(s) and Scientific Research Publishing Inc. This work is licensed under the Creative Commons Attribution International License (CC BY 4.0).

http://creativecommons.org/licenses/by/4.0/

\begin{abstract}
We study the nonlinear parabolic equations for travelling wave solutions of Burger's equations. The purpose of the present work is to study various types of Burger's equations describing waves and those are based on nonlinear equations. We focus on to describe the analytic solution in the special pattern of travelling wave solutions using tan-cot function method. We discuss about inviscid and viscous version of Burger's equation for fluid flow and investigate the effects of internal friction of a fluid via Reynolds number. By changing the velocity amplitude, the nature of flows with shock wave and disturbance are observed. For numerical solutions, the Crank-Nicolson scheme is introduced to establish the wave solutions.
\end{abstract}

\section{Keywords}

Nonlinear PDEs, Tan-Cot Function Method, Travelling Wave Solutions, Burger's Equation, Reynolds Number, Crank-Nicolson Scheme

\section{Introduction}

The wave propagation is one of the important pillar of both linear and nonlinear partial differential equations. A wave is prominently observable which is transported from one segment of the medium to another segment with a recognizable speed of propagation. The mathematical term of wave is a function of the form

$$
u(t, x)=g(x-c t)
$$

where $c$ is a constant known as wave speed and greater than zero; $u$ is a wave function depends on two variables $x$ and $t$. Here $t$ represents the time, the initial 
term translated to the right by $c t$ spatial units.

Many important sectors of sciences such as chemical, physical, economical and biological are introduced by Burger's type nonlinear partial differential equations. It is also noted that in the area of nonlinear modeling of fluid flow and population dynamics the equations pattern like Burger's is rigorously used to present various numerous scientific outcome. Exact solutions to nonlinear differential equations play an important role in physical science since they can provide much more physical information and insight which leads to further applications [1] [2] [3] [4] [5]. Nonlinear wave propagation phenomena of dispersal, dissipation, dispersion, and reaction-diffusion-convection are significant in wave equations. Some of the applications are drawn in the following series:

- Fluid mechanics (water waves, aerodynamics);

- Acoustics (sound waves in air and liquids);

- Elasticity (stress waves, earthquakes);

- Electromagnetic theory (optics, electromagnetic waves);

- Biology (epizootic waves);

- Chemistry (combustion and detonation waves).

To solve the nonlinear equations, there are many analytical and numerical methods in the literature and among them are tan-cot scheme [1] [2], implicit finite difference scheme [3], Hopf-Cole transformation [4], hyperbolic conservation laws [5] [6] [7] [8], sine-cosine method [9] [10] and tanh-sech method [11].

To find the solutions of different patterns of Burgers' equations, variant numerical methods are introduced. Anwar and Ali used tanh and tan-cot schemes for exact complex solutions of some different types of nonlinear partial differential equations [1] [2] whereas Soliman [3] used implicit finite difference scheme. The system of two-dimensional equations is solved by the method called Hopf-Cole transformation [4]. Fletcher used this transformation to give an analytic solution for the system of required equations [4]. In viscous fluid, the approximate theory of flow is used through a shock wave traveling. The persistence of viscous term supports to stop the wave-breaking, and smooth out shock discontinuities and finally the prediction gained a well-behaved and smooth solution. Solutions and characteristics of the Burger's equation with non-zero viscosity, considering shock speed with weak solution are interpreted in [4]-[9]. Several hyperbolic conservation laws with various methods such as Riemann, conservative methods for nonlinear problems which are generalized for the advection equation are discussed in [11]-[16].

In this paper, we discuss the travelling wave solutions of different types of Burger's equations analytically using tan-cot function method. For numerical illustration, we employ finite difference method which is based on Crank-Nicolson scheme to solve one dimensional Burger's equation. Initially, we list out the equations for further analysis. Nonlinear differential equations such as Burger's equation have various types [1] [3] [4] [5] and two well known of them are

1) Travelling wave solution for Burger's equation with viscosity, and 
2) Travelling wave solution of the KdV-Burger's equation.

A simple introduction of these two different forms of Burger's equations is given below:

Consider the Burger equation of the form

$$
\frac{\partial u}{\partial t}=d \frac{\partial^{2} u}{\partial x^{2}}-g(u)
$$

where $d$ is the viscosity of the fluid and $g$ is a nonlinear function of $u$. Introducing the function $g(u)$, the Burger equation can be written in the form of

$$
\frac{\partial u}{\partial t}=d \frac{\partial^{2} u}{\partial x^{2}}-u \frac{\partial u}{\partial x}
$$

here $u$ is the fluid density, $d$ is the viscosity, the spatial variable $x$ and time is $t$. In absence of viscosity, $d=0$, and the Equation (2) becomes

$$
\frac{\partial u}{\partial t}+u \frac{\partial u}{\partial x}=0
$$

which is a non-viscid Burger's equation and the travelling wave solution does not exist. The inviscid (non-viscid) Burgers' equation is a quasilinear wave conservation equation. The analytic traveling wave solution of this equation is an implicit relation that provided characteristics and do not intersect. If the characteristics do intersect, a classical solution to this equation does not exist and leads to the formation of a shock wave. For smooth initial data, the solution satisfied the condition $1+t G^{\prime}(s) \not \equiv 0$ is always satisfied for sufficiently small time $t$, where $G(s)$ is the solution. Otherwise, the solution develops a singularity (discontinuity) and arise the shock wave.

Let us now consider the $\mathrm{KdV}$-Burger's equation of the form

$$
\frac{\partial u}{\partial t}+a u \frac{\partial u}{\partial x}-d \frac{\partial^{2} u}{\partial x^{2}}+k \frac{\partial^{3} u}{\partial x^{3}}=0
$$

which is also known as Korteweg-de Vries Burger's equation and here $a$ and $k$ are arbitrary constants and $d$ is the viscosity. If $a=1$ and $k=0$ then the Equation (4) turn to the Burger's equation.

In the following section, we will discuss the tan-cot function method to get the exact solution of Burger's equation.

\section{Tan-Cot Function Method}

For travelling wave solution of Burger's equation, we used the tan-cot function method as introduced in [1] [2]. The key idea of this method is to discuss in the following steps. Consider the nonlinear partial differential equation in the following form [15] [16]

$$
F\left(u, \frac{\partial u}{\partial t}, \frac{\partial u}{\partial x}, \frac{\partial u}{\partial y}, \frac{\partial^{2} u}{\partial t^{2}}, \frac{\partial^{2} u}{\partial x^{2}}, \cdots\right)=0
$$

where $u(t, y, x)$ is a travelling wave solution of (5). Now using the travelling wave transformation, 


$$
u(t, y, x)=G(s)
$$

where $s=m x+y+n t$. Here $m$ and $n$ are real constant and transformation gives us following changes

$$
\frac{\partial}{\partial t}=n \frac{\mathrm{d}}{\mathrm{d} s}, \frac{\partial}{\partial x}=m \frac{\mathrm{d}}{\mathrm{d} s}, \frac{\partial}{\partial y}=\frac{\mathrm{d}}{\mathrm{d} s} .
$$

By integrating Formula (7) with respect to $s$, a non-linear partial differential Equation (5) transforms to a nonlinear ordinary differential equation such that

$$
Q\left(G, G^{\prime}, G^{\prime \prime}, G^{\prime \prime \prime}, \cdots\right)=0 .
$$

As an instant example, integrate the KdV Burger's equation, (4) with respect to dummy variable $s$ instead of $x$ produces the following differential equation:

$$
\frac{\mathrm{d}^{2} G}{\mathrm{~d} s^{2}}+e_{1} \frac{\mathrm{d} G}{\mathrm{ds}}+e_{2} G^{2}+e_{3} G=e_{0}
$$

where $e_{i},(1=1,2,3)$ to be determined and the integral constant $e_{0}$.

The derivative terms of Equation (8) can be reduced by using integration while the integration constants are negligible and we will obtain the solutions of many nonlinear equations represented by the form

$$
G(s)=\alpha \tan ^{\delta}(\mu s) \text { or } G(s)=\alpha \cot ^{\delta}(\mu s) \text { for all }|s| \leq \frac{\pi}{2 \mu}
$$

where $\alpha, \mu, \delta$ are parameters and to be determined and $\mu$ is known as a wave number.

Remark 1. To get a function $G(s)$ from the ordinary differential Equation (8), the key principle is to integrate this formula as long as all terms contain derivatives and setting the constant of integration to be zero.

First we can write their solutions and derivatives using tan function such that

$$
\left\{\begin{array}{l}
G(s)=\alpha \tan ^{\delta}(\mu s) \\
\frac{\mathrm{d} G}{\mathrm{~d} s}=\alpha \delta \mu\left[\tan ^{\delta-1}(\mu s)+\tan ^{\delta+1}(\mu s)\right] \\
\frac{\mathrm{d}^{2} G}{\mathrm{~d} s^{2}}=\alpha \delta \mu^{2}\left[(\delta-1) \tan ^{\delta-2}(\mu s)+2 \delta \tan ^{\delta}(\mu s)+(\delta+1) \tan ^{\delta+2}(\mu s)\right]
\end{array}\right.
$$

Similarly, for cot function, we have

$$
\left\{\begin{array}{l}
G(s)=\alpha \cot ^{\delta}(\mu s) \\
\frac{\mathrm{d} G}{\mathrm{~d} s}=\alpha \delta \mu\left[\cot ^{\delta-1}(\mu \mathrm{s})+\cot ^{\delta+1}(\mu \mathrm{s})\right] \\
\frac{\mathrm{d}^{2} G}{\mathrm{~d}^{2}}=\alpha \delta \mu^{2}\left[(\delta-1) \cot ^{\delta-2}(\mu \mathrm{s})+2 \delta \cot ^{\delta}(\mu \mathrm{s})+(\delta+1) \cot ^{\delta+2}(\mu \mathrm{s})\right] .
\end{array}\right.
$$

Replacing the Equations (10) or (11) in (8) and after balancing the terms of either tan or cot functions, we can solve the resulting system of algebraic equations by using computerized program. This method is defined as tan-cot function method. 
Next, we will explore the tan-cot function method to find the pattern of exact solutions.

\section{Exploration of Burger's Equations with Respect to Travelling Wave Solution}

In this section, we are interested to solve the Burger's type equations as defined in the earlier section for finding the travelling wave solutions.

\subsection{Travelling Wave Solution for Burger's Equation with Viscocity}

Let us recall the equation in (2) and the wave transformation is

$$
u(t, x)=G(s)
$$

where wave variable $s=(x \pm c t)$ and $c$ is the wave speed. At first, we consider the wave variable, $s=(x+c t)$ and after using the transformation Equation (8), which yields the following ordinary differential equation from (2)

$$
c \frac{\mathrm{d} G}{\mathrm{~d} s}=d \frac{\mathrm{d}^{2} G}{\mathrm{~d} s^{2}}-G \frac{\mathrm{d} G}{\mathrm{~d} s}
$$

Integrating Equation (12) once with respect to $s$, we have

$$
c G+\frac{1}{2} G^{2}-d \frac{\mathrm{d} G}{\mathrm{~d} s}=0
$$

Substituting $G(s)$ and $\frac{\mathrm{d} G}{\mathrm{~d} s}$ from (10), the Equation (13) implies

$$
c \tan ^{\delta}(\mu s)+\frac{1}{2} \alpha \tan ^{2 \delta}(\mu s)-d \delta \mu\left[\tan ^{\delta-1}(\mu s)+\tan ^{\delta+1}(\mu s)\right]
$$

Equating the exponents and the coefficients of each pair of the tan functions, we find that

$$
2 \delta=\delta+1 \Rightarrow \delta=1
$$

and we obtain the following relation

$$
\alpha=2 \mu d
$$

Substituting the values of $\delta$ and $\alpha$ in (10), the solution of the Equation (2) is of the form

$$
u(t, x)=2 \mu d \tan (\mu(x+c t))
$$

which is the travelling wave solution of one dimensional Burger's equation.

Figure 1 depicted the physical structure of travelling wave which counts the wave number and distributions. If we use the transformation $u(t, x)=G(s)$, where $s=x-c t$ then Equation (2) reduces to

$$
-c \frac{\mathrm{d} G}{\mathrm{~d} s}+\frac{1}{2} \frac{\mathrm{d} G}{\mathrm{ds}}-d \frac{\mathrm{d}^{2} G}{\mathrm{ds} s^{2}}=0
$$

and on integration with respect to $s$, we obtain 


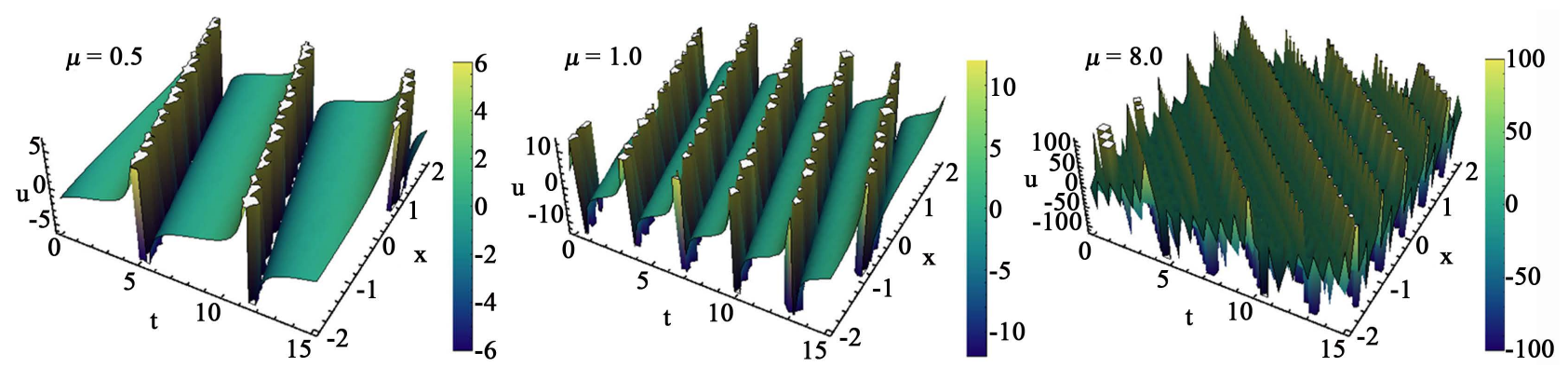

Figure 1. Travelling waves of Burger's equation for $d=c=1$ while (left) $\mu=0.5$, (middle) $\mu=1.0$, and (right) $\mu=8.0$ at time $t=15$ over the domain $x \in[-2,2]$.

$$
\frac{\mathrm{d} G}{\mathrm{~d} s}=\frac{1}{2 d}\left(G^{2}-2 c G-2 A\right)
$$

where $A$ is a constant. For equilibrium points

$$
\frac{\mathrm{d} G}{\mathrm{~d} s}=0 \Rightarrow\left(G-G_{1}\right)\left(G-G_{2}\right)=0
$$

Hence the roots are

$$
G_{1,2}=c \pm \sqrt{c^{2}+2 A}
$$

Let us consider

$$
G_{1}=c-\sqrt{c^{2}+2 A} \text { and } G_{2}=c+\sqrt{c^{2}+2 A}
$$

We observe that $c^{2}>2 A$ and obviously $G_{2}>G_{1}$ then the travelling wave solutions are possible if $\left(G-G_{1}\right)\left(G-G_{2}\right)<0$ for $G_{1}<G<G_{2}$. Now taking integration in (17), we have

$$
\begin{gathered}
\int_{s_{0}}^{s} \frac{\mathrm{d} s}{2 d}=\int \frac{\mathrm{d} G}{\left(G-G_{1}\right)\left(G-G_{2}\right)} \\
\Rightarrow \frac{s-s_{0}}{2 d}=-\frac{1}{G_{2}-G_{1}} \int\left(\frac{1}{G-G_{1}}+\frac{1}{G_{2}-G}\right) \mathrm{d} G \\
\Rightarrow \frac{s-s_{0}}{2 d}=\frac{1}{G_{2}-G_{1}} \ln \left(\frac{G_{2}-G}{G-G_{1}}\right) \\
\Rightarrow\left(G_{2}-G_{1}\right) \frac{s-s_{0}}{2 d}=\ln \left(\frac{G_{2}-G}{G-G_{1}}\right) .
\end{gathered}
$$

After solving this we get

$$
G(s)=\frac{G_{2}+G_{1} \mathrm{e}^{m\left(s-s_{0}\right)}}{1+\mathrm{e}^{m\left(s-s_{0}\right)}}
$$

where

$$
m=\frac{G_{2}-G_{1}}{2 d}>0
$$

Now we can write $G(s)$ as follows 


$$
G(s)=G_{1}+\left(\frac{G_{2}-G_{1}}{2}\right) \frac{2}{1+\mathrm{e}^{m\left(s-s_{0}\right)}} .
$$

After that multiplying and dividing by $\mathrm{e}^{-\frac{m\left(s-s_{0}\right)}{2}}$, we obtain

$$
\frac{2 \mathrm{e}^{-\frac{m\left(s-s_{0}\right)}{2}}}{\mathrm{e}^{\frac{m\left(s-s_{0}\right)}{2}}+\mathrm{e}^{-\frac{m\left(s-s_{0}\right)}{2}}}=1-\frac{\mathrm{e}^{\frac{m\left(s-s_{0}\right)}{2}}-\mathrm{e}^{-\frac{m\left(s-s_{0}\right)}{2}}}{\mathrm{e}^{\frac{m\left(s-s_{0}\right)}{2}}+\mathrm{e}^{-\frac{m\left(s-s_{0}\right)}{2}}}=1-\tanh \frac{m\left(s-s_{0}\right)}{2} .
$$

Initial value $s_{0}$ is not essential and the derivative of $G(s)$ is closer to zero because of smaller $m$ and larger $d$. Moreover, we obtain inviscid Burgers equation which cannot give us continuous travelling waves for $d=0$. So the wave solution is

$$
u(t, x)=\frac{G_{2}+G_{1}}{2}-\frac{G_{2}-G_{1}}{2} \tanh \left(\frac{(x-c t)\left(G_{2}-G_{1}\right)}{4 d}\right)
$$

This solution is similar to shock wave profile because of joining the asymptotic states of $G_{2}$ and $G_{1}$ with the boundary conditions

$$
\begin{gathered}
u(t,-\infty)=G_{2}, \quad t \in[0, \infty), \\
u(t,+\infty)=G_{1}, \quad t \in[0, \infty) .
\end{gathered}
$$

\subsection{Numerical Solutions for Low to High Reynolds Number}

For numerical schematics, we choose finite-difference method based on Crank-Nicolson implicit time differencing [14] [15] [16]. At first we consider the Equation (2) and have to find the dimensionless form of this equation. For obtaining the dimensionless equation of (2), let

$$
\bar{x}=\frac{x}{l}, \quad \bar{u}=\frac{u}{u_{0}}, \quad \bar{t}=\frac{u_{0}}{l} t
$$

where $u_{0}$ and $l$ be the length of velocity amplitude and the calculation area (domain). Hence the non-dimensional form of Equation (2) is

$$
\frac{\partial \bar{u}}{\partial \bar{t}}+\bar{u} \frac{\partial \bar{u}}{\partial \bar{x}}=\frac{d}{u_{0} l} \frac{\partial^{2} \bar{u}}{\partial \bar{x}^{2}}
$$

If we consider $R e=\frac{u_{0} l}{d}$ as a Reynolds number and ignore “-” we can write the above equation as follows:

$$
\frac{\partial u}{\partial t}+u \frac{\partial u}{\partial x}=\frac{1}{R e} \frac{\partial^{2} u}{\partial x^{2}}
$$

Here the limit conditions are periodic and initial condition at $t=0$ are written as $u(0, x)=u_{0} \sin \left(\frac{2 \pi x}{l}\right)$ with the dimensionless variables $\bar{u}(0, \bar{x})=\sin (2 \pi \bar{x})$. We can observe that the high-speed fluid catches up with the slow-moving one so that to create a velocity break. This observation is known as shock. If we declare the disturbance as $u(0, x)=u_{0} \sin \left(\frac{2 \pi x}{l}+\pi\right)$, the 
slope would have decreased. As we say at first for numerical schematics of this dimensionless Equation (21), we use Crank-Nicolson method for simulation investigation. We can elaborate these partial terms of (21) and obtain

$$
\left(\frac{\partial u}{\partial t}\right)_{i}^{j}+\frac{1}{2}\left[\left(\frac{\partial f}{\partial x}\right)_{i}^{j}+\left(\frac{\partial f}{\partial x}\right)_{i}^{j+1}\right]=\frac{1}{2 R e}\left[\left(\frac{\partial^{2} u}{\partial x^{2}}\right)_{i}^{j}+\left(\frac{\partial^{2} u}{\partial x^{2}}\right)_{i}^{j+1}\right]
$$

where $f=\frac{u^{2}}{2}$ and expanding the term $\left(\frac{\partial f}{\partial x}\right)_{i}^{j+1}$, we have

$$
\left(\frac{\partial f}{\partial x}\right)_{i}^{j+1}=\left(\frac{\partial f}{\partial x}\right)_{i}^{j}+K\left[\frac{\partial}{\partial t}\left(\frac{\partial f}{\partial x}\right)\right]_{i}^{j}+O\left(K^{2}\right)
$$

Similarly for other terms and using the notations $\Delta t=K$ and $\Delta x=h$, finally Equation (22) becomes

$$
\begin{aligned}
& \frac{u_{i}^{j+1}-u_{i}^{j}}{K}+\frac{\left(\frac{u^{2}}{2}\right)_{i+1}^{j}-\left(\frac{u^{2}}{2}\right)_{i-1}^{j}}{2 h}+\frac{u_{i+1}^{j}\left(u_{i+1}^{j+1}-u_{i+1}^{j}\right)-u_{i-1}^{j}\left(u_{i-1}^{j+1}-u_{i-1}^{j}\right)}{4 h} \\
& =\frac{1}{2 \operatorname{Re}}\left[\frac{u_{i+1}^{j}-2 u_{i}^{j}+u_{i-1}^{j}}{h^{2}}+\frac{u_{i+1}^{j+1}-2 u_{i}^{j+1}+u_{i-1}^{j+1}}{h^{2}}\right]
\end{aligned}
$$

Remark 2. Due to some limitations over Explicit method, mainly regarding convergence and stability, the another scheme of Finite-difference method is Crank-Nicolson method. The most common features of this method are as follows:

1) Implicit method, unconditionally convergent and stable;

2) The method derived by introducing a fictitious time level at $\left(j+\frac{1}{2}\right)$; and

3) Truncation error of order is $O(\Delta t)^{2}+O(\Delta x)^{2}$, and hence less computation cost.

We can nicely observe the simulation part of this calculation using MATLAB program and some graphical representation. It is noted that everywhere in the numerical simulations, we consider $h=K=0.1$ unless notified the different values.

In both left and right diagrams of Figure 2, let us consider the very small Reynolds number compared to later one and the shock creates and disturbance is erased as soon as it becomes visible. Here $R e=0.1$ and $R e=0.25$ are the values of the Reynolds number and the maximum velocity amplitude is 1.0.

Figure 2 demonstrates the shock waves which appears for viscosity, $d=10$ in the left figure while $d=4$ for the rest one, respectively, where time scales are equal in both figures.

The similar scenarios' are observed in Figure 3 for large Reynolds number ( $R e=10 \& 100)$ compared to Figure 2 although the amplitude structures change its directions and density.

The illustration in Figure 4 (left) establishes that the shock arises and the viscosity is too small ( $d=0.005)$ than the previous two illustrations. The viscosity makes the difference of velocity decrease till there is no break. The 

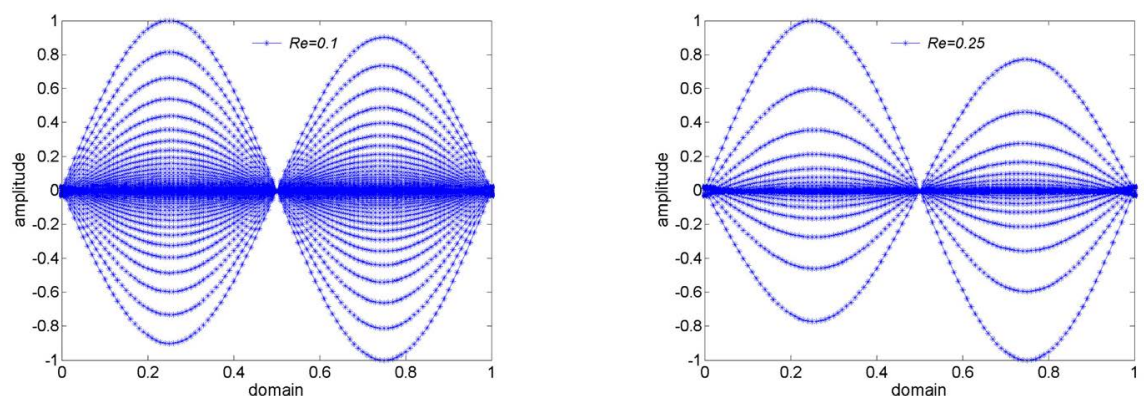

Figure 2. The profile of the solution of Burger's equation for small Reynolds number (left) $R e=0.1$ and (right) $R e=0.25$ at time $t=20$ over the domain.
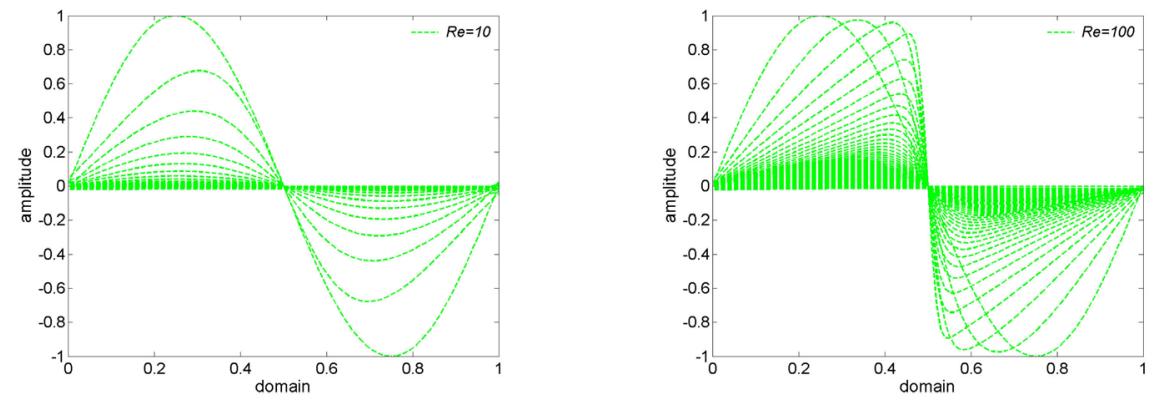

Figure 3. Solutions profile of Burger's equation for Reynolds number (left) $R e=10$ and (right) $R e=100$ at time $t=20$ over the domain.
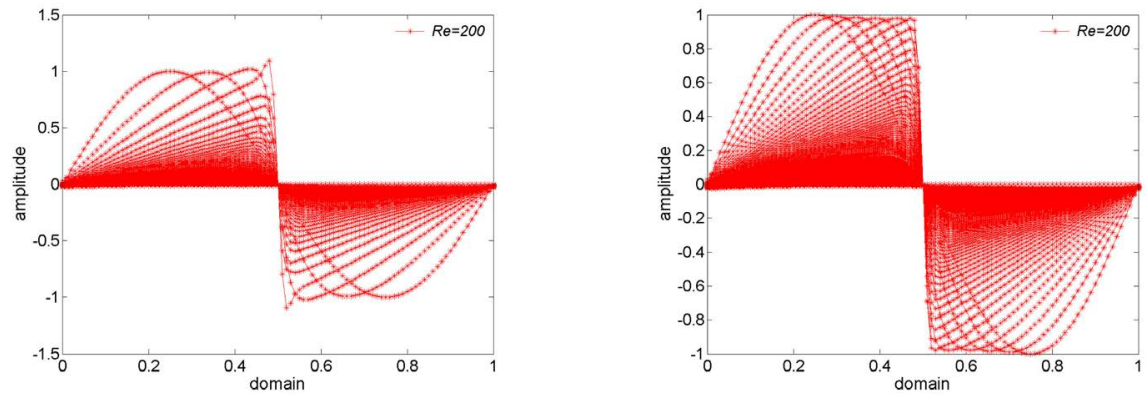

Figure 4. The profile of the solution of Burger's equation for large Reynolds number (left) $R e=200$ and (right) $R e=200$ with time scale $K=0.01$ at time $t=20$ over the domain.

disturbance is increased highly for viscosity which was too small in earlier diagrams, for example in Figure 2 and Figure 3. It creates oscillations around the shock.

If we increase velocity amplitude as $u_{0} \frac{\mathrm{d} t}{\mathrm{~d} x}=1$, we can see the following graphical scenario depicted in Figure 4 (right). The graphical illustration of Figure 4 (right) is similar to Figure 3 (left, right) while $K=0.01$, the time scale is small, even the Reynolds number is equal and $R e=200$ in both figures of 4 . We get similar behavior of flow if we increase the velocity amplitude for $d=0.01$ which is large at time $t=20$. Shock arises and the disturbance is removed. 
Finally in Figure 5, the viscosity is smaller than the previous diagrams for $u_{0} \frac{\mathrm{d} t}{\mathrm{~d} x}=1$. The shock tends to appear and the disturbance is increasing for decreasing of viscosity. More specifically, in Figure 5 (right), we take the value of viscosity $d=0.0003$ which is too small than Figure 5 (left) and notice that the following schematics time scale is still similar.

Investigating those results, we can say that bigger values of viscosity erase the disturbance in the flow. That is, the disturbance has no more issue, if the viscosity is huge. On the other hand, the smaller the viscosity is, the shock appears and the faster disturbance has more effect. So we have concluded that shock arises for small values of viscosity and produces disturbance as oscillations and also it creates sooner when velocity amplitude is bigger.

\section{Travelling Wave Solution of the KdV-Burger's Equation}

We can similarly able to find the travelling wave solutions of KdV-Burger's equation which is described in this portion shortly using only tan-cot function method [1] [2]. Consider the Equation (4), where $a, b$ and $d$ are arbitrary constants. In order to solve (4) by tan-cot function method, let the wave transformation is

$$
u(t, x)=G(s)
$$

where $s=(x+c t)$ and $c$ is the wave speed.

After using the transformation Equation (8), we obtain the ordinary differential equation from (4) such that

$$
c \frac{\mathrm{d} G}{\mathrm{~d} s}=-b \frac{\mathrm{d}^{3} G}{\mathrm{~d} s^{3}}+d \frac{\mathrm{d}^{2} G}{\mathrm{~d} s^{2}}-a G \frac{\mathrm{d} G}{\mathrm{~d} s}
$$

Integrating Equation (24) with respect to $s$, we have

$$
c G+\frac{1}{2} a G^{2}-d \frac{\mathrm{d} G}{\mathrm{~d} s}+b \frac{\mathrm{d}^{2} G}{\mathrm{ds} s^{2}}=0
$$

Then substitute $G(s)$ and $\frac{\mathrm{d} G}{\mathrm{~d} s}$ from (10), the Equation (25) translated to
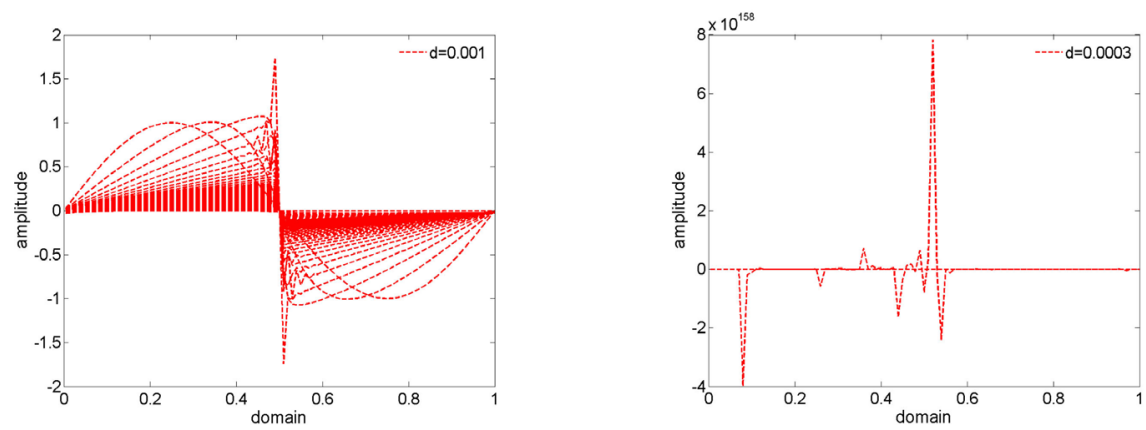

Figure 5. The profile of the solution of Burger's equation for very low viscosity of the fluid (left) $d=0.001$ and (right) $d=0.0001$ at time $t=20$ over the domain. 


$$
\begin{aligned}
& c \tan ^{\delta}(\mu s)+\frac{1}{2} a \alpha \tan ^{2 \delta}(\mu s) \\
& -d \delta \mu\left[\tan ^{\delta-1}(\mu s)+\tan ^{\delta+1}(\mu s)\right] \\
& +b \delta \mu^{2}\left[(\delta-1) \tan ^{\delta-2}(\mu s)+2 \delta \tan ^{\delta}(\mu s)\right. \\
& \left.+(\delta+1) \tan ^{\delta+2}(\mu s)\right]=0 .
\end{aligned}
$$

Equating the exponents and the coefficients of each pair of the tan functions, we find that

$$
2 \delta=\delta+1 \Rightarrow \delta=1
$$

For $\delta=1$, we obtain the following relations from Equation (26)

$$
\left\{\begin{array}{l}
c+2 b \mu^{2}=0 \\
\frac{1}{2} a \alpha-\mu d=0
\end{array}\right.
$$

and it is easy to find the following solutions

$$
c=-2 b \mu^{2} \quad \& \quad \alpha=\frac{2 \mu d}{a}
$$

Substituting the values of $c$ and $\alpha$ in (10), the exact solution of the Equation (4) is of the form

$$
u(t, x)=\frac{2 \mu d}{a} \tan \left(\mu\left(x-2 b \mu^{2} t\right)\right)
$$

which is the travelling wave solution of KdV-Burgers equation.

It is remarked that the next two figures (Figure 6 \& Figure 7) show the significance of different parametric values on exact travelling wave solutions.

The travelling wave solutions pattern are displayed in Figure 6 due to the effect of wave number parameter $\mu$ and for the fixed constants $d=1, a=b=0.1$. It is seen that the travelling waves are visible with the increasing values of $\mu$.

Similarly, Figure 7 shows the effect of constant $d$ when all the parameters are unchanged. It is remarked that wave length is too small and number of wave is easily countable as long as $d$ is very small, see 7 (left) and simultaneously the wave number and length are increasing for larger values of $d$.
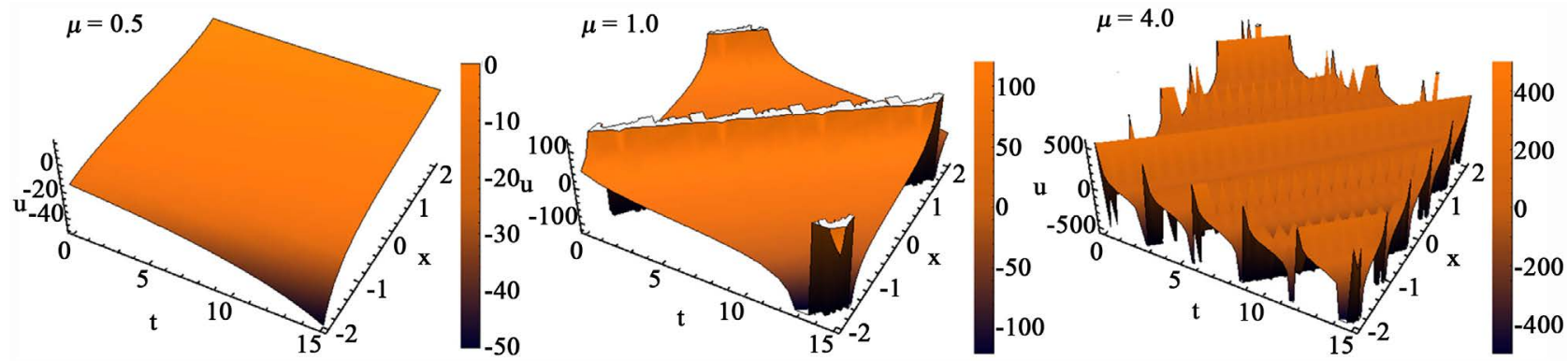

Figure 6. Travelling waves of KdV's equation for $d=1, a=b=0.1$ while (left) $\mu=0.5$, (middle) $\mu=1.0$ and (right) $\mu=4.0$ at time $t=15$ over the domain $x \in[-2,2]$. 


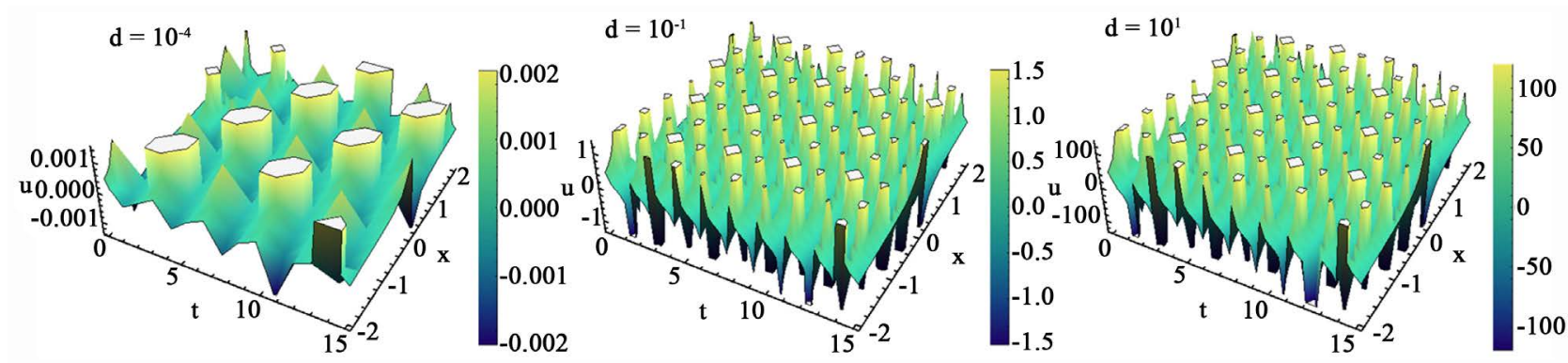

Figure 7. Travelling waves of KdV's equation for $\mu=a=b=1.0$ while (left) $d=10^{-4}$, (middle) $d=10^{-1}$ and (right) $d=10^{1}$ at time $t=15$ over the domain $x \in[-2,2]$.

\section{Conclusion}

In this study, the Burger's equation is solved by using tan-cot function method. Numerically we present the effect of viscosity on amplitude that corresponds to the Reynolds number. The Burger's equation would shock up and tend to break without the presence of viscous terms. Mainly viscous term helps to suppress this breaking effect by countering the nonlinearity and the larger viscosity creates the smaller disturbance. Some exact solutions are presented graphically to observe the travelling waves.

\section{Acknowledgements}

We thank the Editor and the anonymous referees for their comments and suggestions to rich the manuscript.

\section{Conflicts of Interest}

Authors have declared that no competing interests exist.

\section{References}

[1] Jawada, A.J.M. and Ali, Y.S. (2013) New Travelling Waves Solutions for Solving Burger's Equations by Tan-Cot Function Method. International Journal of Computer Engineering Research, 3, 30-35.

[2] Jawad, A.J.M. (2012) New Exact Solutions of Nonlinear Partial Differential Equations Using Tan-Cot Function Method. Studies in Mathematical Sciences, 5, 13-25.

[3] Soliman, A.A. (2000) New Numerical Technique for Burger's Equation Based on Similarity Reductions. International Conference on Computational Fluid Dynamics, Beijing, 17-20 October 2000, 559-566.

[4] Fletcher, J.D. (1983) Generating Exact Solutions of the Two-Dimensional Burger's Equations. International Journal for Numerical Methods in Fluids, 3, 213-216. https://doi.org/10.1002/fld.1650030302

[5] Wazwaz, A.M. (2006) Two Reliable Methods for Solving Variants of the KdV Equation with Compact and Noncompact Structures. Chaos Solitons Fractals, 28, 454-462. https://doi.org/10.1016/j.chaos.2005.06.004

[6] Parkes, E.J. and Duffy, B.R. (1998) An Automated Tanh-Function Method for Finding Solitary Wave Solutions to Nonlinear Evolution Equations. Computer Physics Communications, 98, 288-300. 
https://doi.org/10.1016/0010-4655(96)00104-X

[7] El-Wakil, S.A. and Abdou, M.A. (2007) New Exact Travelling Wave Solutions Using Modified Extended Tanh-Function Method. Chaos Solitons Fractals, 31, 840-852. https://doi.org/10.1016/j.chaos.2005.10.032

[8] Fan, E. (2000) Extended Tanh-Function Method and Its Applications to Nonlinear Equations. Physics Letters A, 277, 212-218. https://doi.org/10.1016/S0375-9601(00)00725-8

[9] Alquran, M., Al-Khaled, K. and Ananbeh, H. (2011) New Soliton Solutions for Systems of Nonlinear Evolution Equations by the Rational Sine-Cosine Method. Studies in Mathematical Sciences, 3, 1-9.

[10] Yusufoglu, E. and Bekir, A. (2006) Solitons and Periodic Solutions of Coupled Nonlinear Evolution Equations by Using Sine-Cosine Method. International Journal of Computer Mathematics, 83, 915-924. https://doi.org/10.1080/00207160601138756

[11] Inc, M. and Ergut, M. (2005) Periodic Wave Solutions for the Generalized Shallow Water Wave Equation by the Improved Jacobi Elliptic Function Method. Applied Mathematics E-Notes, 5, 89-96.

[12] Xia, T.C., Li, B. and Zhang, H.Q. (2001) New Explicit and Exact Solutions for the Nizhnik-Novikov-Vesselov Equation. Applied Mathematics E-Notes, 1, 139-142.

[13] Barenblatt, G.I. (1996) Scaling, Self-Similarity and Intermediate Asymptotic. Cambridge University Press, Cambridge. https://doi.org/10.1017/CBO9781107050242

[14] LeVeque, R.J. (1998) Numerical Methods for Conservation Laws. Birkhauser, Basel, Boston, Berlin.

[15] Evans, L.C. (1998) Partial Differential Equations. AMS, Providence.

[16] Smith, G.D. (1985) Numerical Solutions of Partial Differential Equations: Finite Difference Methods. Oxford Applied Mathematics and Computing Science Series. 